\title{
Geographical disparities in access to cancer management and treatment services in England
}

\begin{abstract}
This study seeks to examine the extent to which cancer services are geographically located according to cancer incidence, and assess the association with cancer survival. We identified hospital sites serving English PCTs (Primary Care Trusts) with the management and treatment of breast, lung and colorectal cancer. Geographical access was estimated as travel time in minutes from LSOAs (Lower Super Output Areas) to the nearest hospital site and aggregated to PCT level. Correlations between PCT level mean travel times and cancer cases were estimated using Spearman's rank correlation. Associations between PCT level mean travel times and cancer relative survival rates were estimated using linear regression with adjustment for area deprivation and for a PCT level measure of the reported ease of obtaining a doctor's appointment. We found that cancer services tended to be located farther from areas with more cancer cases, and longer average travel times are associated with worse survival after adjustment for age, sex, year and area deprivation. This suggests that geographical access to cancer services remains a concern in England.
\end{abstract}

\section{Introduction}

Equity in access to healthcare is an important policy objective in England. The NHS was founded on the principle that services would be available to everyone and would be free at the point of delivery (NHS, 2015). Equity is also embedded in the operating model for the commissioning of specialised services, where NHS England seeks to provide consistent services to all regardless of location (NHS England, 2012). The provision of equitable cancer services is dependent on how they are organised. In England, service configuration since the 1990s has been based on the Calman and Hine report that recommended high quality and also accessible cancer care (Calman and Hine, 1995). The report stated that, 'All patients should have access to a uniformly high quality of care as close to the patient's home as possible' and that 'services should be planned to minimise travelling times whilst maintaining the highest standards of specialist care' (Calman and Hine, 1995, p.6). These recommendations have been endorsed by consecutive Governments with particular attention paid to improving quality by establishing specialised cancer centres (Department of Health, 2000; Haward, 2006). Indeed, some improvements in cancer survival in England have been attributed to this increase in specialisation (Haward, 2006; Oliphant et al., 2013).

The Calman-Hine recommendations also introduced a dilemma with regards to centralisation of services that ensures all patients have access to specialist care without having to travel too far for it (Munro, 2001). Healthcare providers and policymakers are thus faced with the substantial challenge of delivering geographically equitable cancer services within the constraints of finite healthcare resources and in the face of rising cancer incidence rates. Some geographical inequalities in access are inevitable (Gatrell and Wood, 2012) because certain populations, such as rural residents, will always need to travel farther to access specialist services. Inequalities in access are however unacceptable when they lead to avoidable disadvantages in health, and when they disproportionally affect those most in need (Gatrell and Wood, 2012).

Access issues are felt more acutely by those with the greatest need for healthcare, such as patients with chronic conditions who require regular hospital visits, those with lowest mobility such as elderly or disabled patients and also the most deprived (Mungall, 2005; Bentham and Haynes, 1985). Poor access is also known to amplify the effect of deprivation, whereby patients with the longest travel times and also in the most deprived areas are least likely to have a histological cancer diagnosis and optimal treatment (Crawford et al., 2009). In the UK, studies using individual level data have shown a negative association between travel to hospital and uptake for cancer treatment (Jones et al., 2008; Lau et al., 2013) and increased odds of diagnosis at death (Jones et al., 2010). Longer distance to specialist cancer centres has also been associated with higher cancer stage at diagnosis 
(Campbell et al., 2001) and with poorer survival (Campbell et al., 2000). These findings have been replicated outside the UK; in France, road distance to the nearest cancer centre was associated with worse survival (Dejardin et al., 2008). Studies that have employed alternative measures of healthcare accessibility have reached similar conclusions. For instance, one North American study used a derivative of the gravity model to capture the availability (supply) as well as the attractiveness (demand) of services (Wang, 2006), the study demonstrated an association between poor geographical access and advanced cancer stage (Wang et al., 2008).

Our study builds on the existing evidence by investigating geographical inequities in access at a population level. We define geographical access as travel times in minutes from area of residence to the nearest hospital for cancer management or treatment. We have applied the definition of equity that is most generally accepted by policy makers; equal access for equal need (Oliver and Mossialos, 2004; Allin et al., 2007), with 'need' being the capacity to benefit where there is an effective and acceptable intervention to improve or prevent ill health (Matthew G, 1971; Wright et al., 1998). Healthcare need at a population level can be measured by the 'level of ill health' (Allin et al., 2007) and epidemiological measures such as prevalence or incidence can be used to describe 'how much of it there is' and 'where it is located' (Acheson, 1978; Williams and Wright, 1998).

Geographical inequities in access will be determined where areas with higher need also have poorer access to cancer services. Additionally, geographical access will be associated with relative survival rates to determine whether areas with poor access also have the worst outcomes. Lung, breast and colorectal cancers are among the commonest cancers totalling to about $40 \%$ of all cancer incidence in the England and amounting to approximately 120,000 cases annually (Cancer Research UK, 2016a). Treatment for these requires access to MDT, chemotherapy and radiotherapy, and so they are appropriate cancer sites for this work.

\section{Methods and Materials}

The study has a cross sectional ecological design, with measurements and inferences made at the level of NHS Primary Care Trust (PCT) area, as this was the scale at which data was available. It was not possible to obtain data on lower geographies for this study.

We used multiple sources to obtain information on the location of cancer hospital sites. In England, this information is collected by the National Peer Review Team (National Peer Review Programme, 2013) which holds details on the location of hospital sites that provide cancer treatment (chemotherapy and radiotherapy), and sites providing cancer management via multidisciplinary teams (MDTs). In order to account for the fact that patients in some parts of England may receive treatment in Wales and Scotland, we obtained similar Welsh data from the Welsh Health Directory (NHS Wales Informatics Services). At the time of the analysis, the Welsh Health Directory had complete information on the North Wales Cancer Network, but information was incomplete on the South Wales Cancer Network. Missing information was supplemented by Freedom of Information (FOI) requests from the Welsh Health Boards that are part of the South Wales Cancer Network. We also received data from the Information Services Division Scotland (Information Services Division, 2015) on hospital sites located in the southern Scotland Health Boards that may serve some English patients; Dumfries and Galloway, Borders, Ayrshire and Arran, Lanarkshire, Lothian and Greater Glasgow and Clyde. The Scottish data did not have information on MDT presence, and therefore hospitals offering chemotherapy were used as a proxy for presence of a MDT.

All the identified hospital sites were assigned a unique postcode, this is a geographical reference point in the U.K used to identify postal delivery areas (Office of National Statistics, 2015a). There are about 1.8 million postcodes and each have approximately 15 to 100 single addresses. Larger addresses that receive numerous mail items per day such as hospital sites, are assigned a single postcode and hence are accurately located (Office of National Statistics, 2015a). 
Geographical access was determined as estimated travel time in minutes from all LSOA population weighted centroids in England, to the nearest hospital site offering treatment or management for the specified cancer. LSOAs are small geographic areas in England and Wales that are designed to improve the reporting of small area statistics. There are 32,844 LSOAs in England each with a population range of 1,000 to 3,000 residents (Office of National Statistics, 2015b). A population weighted centroid is a summary reference point at the centre of the population in a geographical unit (Office of National Statistics, 2013b). We used a Geographical Information System (GIS) (ArcGIS 10.3, Esri Inc.) Network Analyst module to estimate travel times from all 2011 LSOA population centroids in mainland England, to all postcodes of the identified hospital sites. The travel times generated at LSOA level were thereafter aggregated to the PCT level as this is the level at which cancer data was available. Aggregation was achieved by summing up all LSOA level travel times in a given PCT and obtaining an average. PCTs were English health administrations at the time of data collection that were responsible for planning and purchasing primary, community and secondary health services. They have a median resident population of 203,000 (Office of National Statistics, 2013c). There were 152 PCTs in England, at the time of this analysis.

The National Radiotherapy Advisory Group (NRAG) recommend travel of no longer than 45 minutes for radiotherapy treatment (National Radiotherapy Advisory Group, 2007) and therefore we used this as an important threshold for radiotherapy treatment. For travel to MDT and Chemotherapy, we used 20 minutes to mark an important threshold as this was the approximate average travel time to hospitals in England during the study period (Department for Transport, 2014). Further, we quantified the proportion of the population in England whose travel may exceed these thresholds using ONS 2009 mid-year population estimates (Office of National Statistics, 2013c; Office of National Statistics, 2013a).

The measure of population need adopted for this analysis was the number of cases of (breast (ICD10 C50), colorectal (ICD-10 C17-21 and C26) and lung (ICD-10 C33-34) cancer in a PCT. This was obtained as a three year average for 2008 - 2010 from the publicly available National Cancer Intelligence Network (NCIN) dataset of newly diagnosed cancer cases per year (National Cancer Intelligence Network, 2010). Primary outcomes were identified as one and five year PCT relative survival rates for each cancer, also obtained from the NCIN public dataset (National Cancer Intelligence Network, 2014a). These relative survival rates were estimated nationally by NCIN using the actuarial method that divides observed with expected survival rates to give a population level relative survival rate (Parkin and Hakulinen, 1991; National Cancer Intelligence Network, 2014b). The 'observed one year survival rates' were estimated as the number of persons diagnosed with the specified cancer between 2010 and 2012 with mortality follow up to the end of 2013. The 'observed five year survival rates' were also estimated in the same way but for patients who had a diagnosis between 2002 and 2004 and followed up to 2009. The 'expected survival rates' were based on the population life tables matched by age, sex and period of observation (Parkin and Hakulinen, 1991; National Cancer Intelligence Network, 2014b).

Every effort was made to match the timescale covered by the estimated travel times with that of the incidence and survival data. This was achieved for travel to radiotherapy as there were fewer sites involved. Thus, travel time to radiotherapy was restricted to sites in operation before 2010 to match the incidence data. The three new radiotherapy hospital sites that opened after 2010 were excluded from the analysis; Bracknell Clinic, Fazackerley site of Clatterbridge Cancer Centre and The Christie at Royal Salford. For the regression analysis, we also used hospital sites identified as above because the cancer cases used to estimate relative survival rates would have been diagnosed in the same decade as the incidence data; 2010 - 2012 for 1 year survival and 2002 - 2004 for 5 year survival. We were however unable to discern MDT and chemotherapy sites by year of operation and therefore utilised the most recent data at the time of the analysis (November 2014). The discrepancies between the time of data collection and study period might have a slight effect on the results, but we think this effect is not substantial because English hospital sites would not have changed substantially during this decade. 
Deprivation and ease of obtaining a general practitioner (GP) appointment were identified as potential confounders as they are independently associated with cancer survival. PCT level deprivation data was obtained from the English Index of Multiple Deprivation (IMD) (Department for Communities and Local Government, 2010). Good access to primary care is important in the early diagnosis and later prognosis of cancer and has previously been associated with lower rates of emergency admissions to secondary care (Cowling et al., 2013). Ease of obtaining a GP appointment was measured as the percentage of patients in a GP practice who were able to get an appointment to see or speak to a health professional during their most recent appointment, without the need to call back closer to or on the day they wanted an appointment. This is information is collected on an annual basis by the national GP Patient Survey of 2.75 million adults registered with a GP in England (NHS England, 2012-2013).

\section{Statistical analysis was conducted on using Stata (Version 13.1; StataCorp, College}

Station, TX, USA). Spearman's rank correlation was used to correlate the unadjusted PCT cancer cases (need) with the estimated mean PCT travel times (access). Linear regression was used to examine the association between cancer relative survival rates and PCT level mean travel times, adjusting for age, sex, year, area deprivation and ease of GP access. Models were tested to ensure they met the following assumptions of the linear regression models; linearity, normality and homoscedasticity of the residuals. Sensitivity analysis was performed by modelling travel times both as a continuous measure and a grouped measure and examining any changes in the results.

Standard errors of the relative survival rates were estimated to help determine the extent to which they were influenced by sampling variation (Ederer et al., 1961). A p-value $(<=0.05)$ was used to identify statistical significance in all analyses.

\section{Results}

Table 1 shows the distribution of the estimated mean PCT travel times. Radiotherapy was provided at the fewest number of hospital sites and therefore incurred the longest journey times with a mean of 23.7 minutes. Table 2 groups the PCTs in England by the mean travel times to radiotherapy hospital sites. It also gives the average deprivation score and estimated population in the different travel time groups. Using the mid-2009 PCT population, an estimated 3 million (5.72\%) people and over 6,000 breast, colorectal and lung cancer patients in England lived more than 45 minutes from a radiotherapy facility during the study period. Figure 1 demonstrates how these mean travel times to radiotherapy are distributed in England at (a) LSOA and (b) PCT levels. For the purpose of brevity, only the travel to radiotherapy sites are provided.

The number of cancer cases in a PCT across the three cancer types were positively and significantly correlated with longer PCT mean travel times to all hospital sites $(p<0.01)$ (Table 3).

Table 1 - Number of cancer services and estimated PCT mean travel times in England, Wales and Scotland*

\begin{tabular}{lccccccc}
\hline Type of service & $\begin{array}{c}\text { Number of } \\
\text { sites }\end{array}$ & \multicolumn{6}{c}{ Travel times in minutes PCT } \\
& & Lowest & Mean & Highest & $\mathbf{2 5}^{\text {th }}$ Quartile & Median & $\mathbf{7 5}^{\text {th }}$ Quartile \\
\hline Breast MDT & 214 & 4.0 & 13.5 & 35.2 & 9.5 & 12.4 & 17.1 \\
Colorectal MDT & 226 & 3.8 & 13.3 & 35.2 & 9.1 & 12.2 & 16.3 \\
Lung MDT & 219 & 3.8 & 13.5 & 35.2 & 8.9 & 12.4 & 16.4 \\
Chemotherapy & 231 & 3.6 & 13.1 & 35.2 & 8.7 & 11.9 & 15.7 \\
Radiotherapy & 61 & 4.0 & 23.7 & 61.0 & 13.8 & 22.4 & 32.0 \\
\hline
\end{tabular}

* Scottish data was obtained from the southern Health Boards only; Dumfries and Galloway, Borders, Ayrshire and Arran, Lanarkshire, Lothian and Greater Glasgow and Clyde. MDT, multidisciplinary team. LSOA, Lower Super Output Area 
Table 2 - Population estimates and cancer cases grouped by travel times to radiotherapy services. Cancer cases are estimated using PCT mid-2009 population estimates and three year annual cancer cases $(2008-2010)$

\begin{tabular}{lllll}
\hline $\begin{array}{l}\text { Travel times to } \\
\text { radiotherapy } \\
\text { services (minutes) }\end{array}$ & $\begin{array}{l}\text { PCTs by } \\
\text { Travel time } \\
\text { categories }\end{array}$ & $\begin{array}{l}\text { PCT Overall IMD } \\
\text { average by Travel } \\
\text { time categories }\end{array}$ & $\begin{array}{l}\text { PCT Population mid } \\
\text { 2009 estimates } \\
\text { (\% of total) }\end{array}$ & $\begin{array}{l}\text { Estimated breast, } \\
\text { colorectal and lung } \\
\text { cancer cases }\end{array}$ \\
\hline $0-14.99$ & 43 & 28.11 & $11,451,308(22.00 \%)$ & 23,647 \\
$15-29.99$ & 65 & 22.26 & $24,010,852(46.12 \%)$ & 49,574 \\
$30-44.99$ & 34 & 21.24 & $13,616,842(26.16 \%)$ & 28,119 \\
45 plus & 8 & 21.36 & $2,979,018(5.72 \%)$ & 6,148 \\
\hline
\end{tabular}

Table 3 - Correlation coefficient of mean PCT mean travel time to cancer services and PCT cancer incidence (number of new cases, annual average for 2008-2010)

\begin{tabular}{|c|c|c|c|c|c|}
\hline $\begin{array}{l}\text { Number of new } \\
\text { cases per year }\end{array}$ & $\begin{array}{l}\text { Breast } \\
\text { MDT }\end{array}$ & $\begin{array}{l}\text { Colorectal } \\
\text { MDT }\end{array}$ & $\begin{array}{l}\text { Lung } \\
\text { MDT }\end{array}$ & Chemotherapy & Radiotherapy \\
\hline Breast & $0.68^{\star *}$ & & & $0.68^{* \star}$ & $0.45^{\star \star}$ \\
\hline $\begin{array}{l}\text { Colorectal } \\
\text { Lung }\end{array}$ & & $0.72^{\star \star}$ & $0.58^{\star *}$ & $\begin{array}{l}0.72^{\star \star} \\
0.58^{\star \star}\end{array}$ & $\begin{array}{l}0.46^{\star *} \\
0.41^{* *}\end{array}$ \\
\hline
\end{tabular}

Figure 1 - Map of mean travel times to radiotherapy services estimated at (a) LSOA and (b) PCT levels. Travel times were calculated from all English LSOA centroids to all radiotherapy hospital sites operating within the study period. The computed travel times were later on aggregated to PCT level.

The test for linearity using the Stata 'nlcheck' tool (Jann, 2008) showed that continuous 'travel time' and 'ease of GP access' variables failed to meet this assumption, which supported the decision to model these variables as categories. The 'Shapiro-Wilk' test for the normality of residuals also showed that some models failed to meet this assumption. However, this does not invalidate the analysis because the inferences in linear regression are robust to violations of normality so long as the sample size is reasonably large (Agresti, 2015; Xin and Su, 2009). Although it is recognised that non-normality does not affect the validity of the models, heteroscedasticity may affect it (Lumley et al., 2002). Four out of eighteen of the fully adjusted models did not meet this homoscedasticity assumption, these four models are for lung cancer access to MDT and Chemotherapy services (Supplementary tables 5 and 6), and hence these findings should be treated with caution. Modelling travel times as a continuous rather than categorical measure, did not alter the conclusions.

Estimated standard errors of the relative survival rates across the three cancer sites, and for both one and five years were generally small compared to their associated estimates, and ranged from 0.004 to 0.049 .

When estimated PCT mean travel times were regressed on cancer relative survival rates, the unadjusted models for breast and colorectal cancers showed that longer travel time was positively associated with longer survival. This was mostly evident for travel to MDTs and chemotherapy services and was statistically significant for five year relative survival rates. For example, for travel to breast and colorectal MDTs, PCTs with the longest mean travel had a significantly higher five year survival rates (breast coefficient $=1.79$; $\mathrm{SE}=0.79 ; \mathrm{t}(148)=2.26 ; \mathrm{p}<0.05$ ) and (colorectal coefficient $=4.10 ; \mathrm{SE}=1.16 ; \mathrm{t}(148)=3.54 ; \mathrm{p}<0.01)$. Longer travel to radiotherapy was also positively associated with breast and colorectal cancer although this only reached statistical significance for the five year breast cancer survival rates $(1.27$; $S E=0.59 ; \mathrm{t}(148)=2.15 ; \mathrm{p}<0.05)$ (Table 4). In contrast, the lung cancer analysis showed that PCTs with the longest mean travel had significantly 
lower one and five year relative survival rates. The rest of the results for MDT and Chemotherapy analysis are presented as on-line supplementary material (Tables 5 and 6) for brevity.

Adjusting for deprivation and ease of GP access revealed a clear survival gradient whereby PCTs with longer mean travel times consistently had poorer relative survival rates. This survival gradient was observed in travel to all services across the three cancers. As an example, Table 4 shows the regression output for the models of survival and access to radiotherapy treatment. The coefficients are percentage point differences in relative survival associated with each explanatory variable. The lung cancer output for example shows that an increase in travel times from the reference category 'less than fifteen minutes' to the highest category ' 45 plus minutes', is associated with a predicted reduction in one year survival by -3.43 percent, $p<0.05$ (before adjusting), -4.02 percent, $p<0.01$ (after adjusting for deprivation only) and -4.60 percent, $p<0.01$ (after adjusting for deprivation and ease of obtaining a GP appointment).

In the lung cancer analysis, the travel - survival association was generally greater for one year than five year relative survival rates. For example, the lung cancer output in Table 4 shows the corresponding predicted reduction in the five year relative survival is -2.40 percent, $p<0.05$ (before adjusting), -2.72 percent, $p<0.05$ (after adjusting for deprivation only) and -2.98 percent, $p<0.01$ after adjusting for deprivation and ease of obtaining a GP appointment. Lastly, we found that the travel to radiotherapy - survival association was greater in colorectal and lung cancer compared to breast cancer (Table 4). 
Table 4 - PCT mean travel times to the nearest radiotherapy site, associated with 1 and 5 year breast, colorectal and lung cancer relative survival rate. The table shows linear regression model outputs using 150 PCTs in England, adjusted for deprivation and ease of obtaining a GP appointment.

\section{Not adjusted for deprivation} or ease of GP appointment

\section{Breast}

CRC

Lung

\section{Adjusted for deprivation}

Breast

CRC

Lung

\section{Adjusted deprivation \& ease of}

GP appointment

\section{Explanatory variables}

Outcome: 1 Year Relative Survival

Travel times to Radiotherapy

$<15$ minutes (reference)

$15-29.9$ minutes

$30-44.9$ minutes

$-0.12$

45 minutes plus

$-1.58^{*}$

$-3.08^{\star *}$

-0.59
-0.30
-0.20

$-1.29^{*}$

$-3.68^{* *}$

$-0.62^{\star *}$

$-0.30$

$-0.98$

-0.98
$-1.31^{*}$
$-2.67^{\star}$

$-1.94^{*}$

Deprivation (IMD)

$-0.08^{* *}$

$-0.17^{\star *}-0.09^{*}$

$-0.09^{* *}$

$-0.14^{* *}$

$-4.60^{* *}$

Ease of obtaining a GP appointment

Quartile 1 - Difficult

Quartile 2

Quartile 3

Quartile 4 - Easy

Intercept (Y)

R-squared

Outcome: 5 Year Relative Survival

$\begin{array}{rr}96.44^{\star \star} & 76.51^{\star \star} \\ 0.02 & 0.01\end{array}$

$34.39^{* *}$

0.10

$98.67 * * 28$
0.28

$81.15^{\star *} \quad 36.85^{\star *}$

$$
-0.27
$$

$-0.50^{*}$

$-0.81^{* *}$

$99.42^{* *}$
0.33

Travel times to Radiotherapy

$<15$ minutes (reference)

$15-29.9$ minutes
$30-44.9$ minutes
45 minutes plus

$\begin{array}{rr}1.27^{\star} & 0.50 \\ 0.77 & -0.05 \\ 1.07 & -0.16\end{array}$

$-1.15^{\star}$

0.13

$-0.56$

$-0.24$

$-1.14-1.44^{\star *}$

$-1.97^{*}-1.68^{\star *}$

0.20

$-2.05-2.72^{* *}$

$-0.55$

-0.84
$-2.03^{*}$

$-0.72-3.49^{*}$

$-0.19^{* *}$

$-0.28^{\star \star}-0.05^{\star}$

$-0.17^{\star *}$

$-0.22^{\star *}$

$-2.98^{\star \star}$

Deprivation (IMD)

Ease of obtaining a GP appointment

Quartile 1 - Difficult

Quartile 2

Quartile 3

Quartile 4 - Easy

Intercept $(Y)$

$84.54^{\star \star}$

$53.62^{* *}$

$9.614^{\star *}$

$89.99 * *$

$61.50^{* *} 11.03^{* *}$

$0.24 \quad 0.10$

0.07

0.29

Numbers show the regression coefficients (standardized $\beta$ ). All relative survival rates are adjusted for age, sex and year. ${ }^{* *} p<0.01,{ }^{*} p<0.05$. 


\section{Discussion}

Our findings show that PCTs with more cases of breast, colorectal and lung cancer also have longer estimated mean travel times to the cancer management and treatment services we mapped. PCTs with longer mean travel times also have poorer relative survival rates after adjustment for area deprivation. These findings highlight the importance of examining inequalities in access to services and should inform NHS England's mandate of ensuring equitable commissioning of specialised services (NHS England, 2012).

We used an area level dataset for this analysis. It is likely that inference at this level will suffer to some extent from ecological fallacy by suggesting that relationships between areas may be related to individuals within them. Nevertheless, area based studies have several advantages, such as contributing to theory and hypothesis generation for future testing (Pearce, 2000). We have used publicly available datasets to highlight issues in access that warrant further investigation using more robust individual level datasets. The study also offers a national perspective on geographical access and augments the evidence previously conducted using smaller regions. Although incidence data is available at lower geographies, confidentiality restrictions meant we were unable to obtain this for the study. Relative survival rates on the other hand have not been computed at lower geographies in England, because the small number of cases and deaths at lower geographies introduces difficulties in standardising by age and sex. Whilst acknowledging that using relatively large PCT geographies conceals variation present at lower geographies, our findings are still informative because commissioning of cancer services in England took place at this scale (Okello et al., 2011).

Using area level data we were able to demonstrate that areas with the poorest access also have the highest cancer cases (Table 3). This may be an indication that services in England may not be located according to population need. One explanation is that cancer services are more likely to be located in cities. This is contrary to the fact that the demand for these services is greater in less urban areas that carry a larger cancer burden due to larger percentage of older people. A programme commissioned by the National Cancer Action Team to model radiotherapy demand in England showed local area variation. Inner London PCTs with younger populations had a lower projected demand by the year 2020; 6,007 fractions per million in comparison to 20,827 fractions per million for more rural PCTs such as Devon (Round et al., 2013). This variation is due to geographical variation in age distribution because older age carries an increased cancer burden (Round et al., 2013).

Associating PCT level mean travel times with cancer survival rates showed that areas with longer travel times to MDT and treatment have the worst cancer survival rates. Our unadjusted models for breast and colorectal cancers showed that areas with longer travel were more likely to have better relative survival rates. However, when we removed the confounding effect of deprivation we found the opposite; areas with longer travel were more likely to have poorer relative survival rates. This is because deprived populations are more likely to live in urban areas where most hospitals are located.

Our findings indicate that travel time - survival association was strongest in lung and colorectal cancers than in breast cancer, this is particularly so for radiotherapy treatment (Table 4). Increasing distance from a cancer centre has been significantly associated with poorer survival in lung cancer (Campbell et al., 2000). Poor survival for those with poorer access may be explained by the increased likelihood of more advanced disease stage at diagnosis (Campbell et al., 2001), but why this might vary by tumour site is less clear. Other research suggests treatment uptake also appears to vary by tumour type; breast, rectal and lung cancers patients are all less likely to receive radiotherapy if they live farther from a radiotherapy site (Jones et al., 2008). Additionally, poor access to chemotherapy for rectal and lung cancer treatment (Jones et al., 2008) and poor access to surgical treatment for lung cancer has been associated with reduced likelihood for these treatments (Jones et al., 2008; Lau et al., 2013; Khakwani et al., 2013). There is also some evidence that geographical inequalities in cancer survival has declined for breast cancer but has persisted for other cancers (Walters et al., 2011), our findings may to some extent support this conclusion. 
We also found that geographical access to radiotherapy had a stronger association with relative survival rates than geographical access to MDT and chemotherapy services. This is most likely because there are fewer sites for radiotherapy than for MDT and chemotherapy. NRAG recommends a threshold of 45 minutes travel for radiotherapy treatment (National Radiotherapy Advisory Group, 2007). Using ONS mid 2009 PCT population estimates and mean PCT travel times, we estimated that nearly $6 \%$ (3 Million) of the population in England lives farther than the recommended 45 minutes. It is likely that this population estimate is greater, this is because analysis at large areas such as PCTs conceals the extent of variation observed at smaller areas. For example, similar analysis at LSOA level showed that an estimated 10\% (5 million) of the population in England lives over 45 minutes from a radiotherapy hospital site.

Surgical operation is the main treatment for colorectal cancer and non-small cell lung cancer (Cancer Research UK, 2016b). In recent decades, the role of radiotherapy in cancer treatment was understated and this resulted to an under-investment of radiotherapy services in the UK (Cancer Research UK, 2009). However, its curative effectiveness is now increasingly being recognised, with nearly $40 \%$ of cured cancer cases being attributed to radiotherapy treatment (National Radiotherapy Advisory Group, 2007). Where used curatively in lung cancer, it is generally reserved in the treatment of inoperable tumours often in elderly patients or those with other multiple morbidities (Louie et al., 2015). It is also used often to treat rectal but not colon cancers, whilst in breast cancer, radiotherapy is used after breast reconstruction and mastectomy (Cancer Research UK, 2016b). A substantial under-provision of radiotherapy services in England has been reported with an estimated $63 \%$ gap between current activity levels and optimal treatment levels, however, improvements to increase provision are underway (National Radiotherapy Advisory Group, 2007). Our findings suggest that good access to radiotherapy treatment may be an important prognostic factor in breast, colorectal and lung cancer.

This study has a number of limitations. It is a cross-sectional study and therefore causality cannot be determined; it is plausible for example that longer travel times might have caused poorer survival because of later diagnosis or inadequate treatment, although no data was available to let us test this. Another limitation is that we used number of cancer cases to indicate population need. It could be argued that the number of cases are only an indicator of the quantity of services needed, and not of the needs of individuals who have cancer. We have used aggregated PCT level data as individual level data or data from smaller geographies was not available at the time of the analysis.

Aggregated data exposes the study to the ecological fallacy meaning the findings at a population level may not be inferred at individual level. Additionally, some of our data sources did not cover the same time period. For example there was no information to identify the location of MDT and chemotherapy hospital sites operating in the period over which the outcomes were measured, although it is unlikely that the geographical provision of services will have changed substantially over the period of this study. Data were not available on mobile chemotherapy units or GP surgeries offering chemotherapy services and so we were unable to include them in our access measures. Another limitation is that PCTs are now obsolete geographies and have been replaced by CCGs (Clinical Commissioning Groups). At the time of analysis it was not possible to obtain cancer specific five year relative survival rates at CCG level.

In conclusion, we have shown that despite equity in access being a key objective in the provision of healthcare, it remains an elusive goal in the provision of cancer services in England. Our findings suggest that longer travel times to services might lead to inadequate treatment and therefore poorer survival. We have also identified some research questions that warrant more in-depth future research. Firstly, colorectal and lung survival appear to be more sensitive to poor geographical access to radiotherapy than breast cancer (Table 4). Secondly, we found that in lung cancer, geographical access issues were more strongly associated with one year over five year survival rates. Thirdly, lung cancer survival appears to be more sensitive to geographical access to chemotherapy and MDT than both colorectal and breast cancer.

Part of the challenge in meeting the equity goal may lie in the lack of a universal definition and monitoring criteria for equity in access (Allin et al., 2007). Another challenge lies in the trade-off that 
results when attempting to meet contrasting goals such as equity and efficiency, with resources that are increasingly scarce. This has been recognised by the recent Cancer Research Taskforce for England that is working to develop a five year cancer survival improvement strategy on behalf of NHS England and other national health agencies (Independent Cancer Taskforce, 2015). The Taskforce has identified a need to revisit the issue of access to services in order to enable improvements in earlier diagnosis and quality of care. It has also recognised the inequitable commissioning and delivery of care, and has urged the tackling of variation to be a priority over the next five years. To achieve this, two recommendations that are relevant to this study have been put forward; an evaluation of evidence to determine whether service configuration of surgery merits further centralisation and, an evaluation of the impact on cancer outcomes of patients living distances from a cancer centre (Independent Cancer Taskforce, 2015).

\section{Conflict of interest}

None

\section{References}

Acheson, R. M. 1978. The definition and identification of need for health care. Journal of Epidemiology and Community Health, 32(1), pp 10-15.

Agresti, A. 2015. Foundations of Linear and Generalized Linear Models, Hoboken, New Jersey: John Wiley \& Sons.

Allin, S., Masseria, C., Sorenson, C., Papanicolas, I. \& Mossialos, E. 2007. Measuring inequalities in access to health care. A review of the indices,

Bentham, G. \& Haynes, R. 1985. Health, personal mobility and the use of health services in rural Norfolk. Journal of Rural Studies, 1(3), pp 231-239.

Calman, K. \& Hine, D. 1995. A Policy Framework for Commissioning Cancer Services. Guidance for Purchasers and Providers of Cancer Services, Health, D. o. (London).

Campbell, N. C., Elliott, A. M., Sharp, L., Ritchie, L. D., Cassidy, J. \& Little, J. 2000. Rural factors and survival from cancer: analysis of Scottish cancer registrations. Br J Cancer, 82(11), pp 1863-1866.

Campbell, N. C., Elliott, A. M., Sharp, L., Ritchie, L. D., Cassidy, J. \& Little, J. 2001. Rural and urban differences in stage at diagnosis of colorectal and lung cancers. Br J Cancer, 84(7), pp 910914.

Cancer Research UK. 2009. Achieving a world-class radiotherapy service across the UK,

Cancer Research UK. 2016a. Cancer cases and rates by country in the UK [Online]. CRUK. Available: http://publications.cancerresearchuk.org/publicationformat/formatstats/dtinccountries.html [Accessed February 2016]. 
Cancer Research UK. 2016b. Your cancer type [Online]. CRUK. Available:

http://www.cancerresearchuk.org/about-cancer/type/ [Accessed August 2015].

Cowling, T. E., Cecil, E. V., Soljak, M. A., Lee, J. T., Millett, C., Majeed, A., Wachter, R. M. \& Harris, M. J. 2013. Access to Primary Care and Visits to Emergency Departments in England: A Cross-Sectional, Population-Based Study. PLoS ONE, 8(6), pp e66699.

Crawford, S. M., Sauerzapf, V., Haynes, R., Zhao, H., Forman, D. \& Jones, A. P. 2009. Social and geographical factors affecting access to treatment of lung cancer. British Journal of Cancer, 101(6), pp 897-901.

Dejardin, O., Bouvier, A. M., Faivre, J., Boutreux, S., De Pouvourville, G. \& Launoy, G. 2008. Access to care, socioeconomic deprivation and colon cancer survival. Alimentary Pharmacology \& Therapeutics, 27(10), pp 940-949.

Department for Communities and Local Government. 2010. English Indices of Deprivation [Online]. Available: https://www.gov.uk/government/statistics/english-indices-of-deprivation-2010 [Accessed June 2015].

Department for Transport. 2014. Average minimum travel time to reach the nearest key services by mode of travel, England, 2007 to 2013 (Table ACS0101) [Online]. Available:

https://www.gov.uk/government/statistical-data-sets/acs01-availability-of-transport-to-keyservices-or-work-among-households [Accessed June 2015].

Department of Health. 2000. The NHS Cancer Plan, a plan for investment, a plan for reform, Department of Health (London).

Ederer, F., Axtell, L. M. \& Cutler, S. J. 1961. The relative survival rate: a statistical methodology. Natl Cancer Inst Monogr, 6(101-21.

Gatrell, A. C. \& Wood, D. J. 2012. Variation in geographic access to specialist inpatient hospices in England and Wales. Health \& Place, 18(4), pp 832-840.

Haward, R. A. 2006. The Calman-Hine report: a personal retrospective on the UK's first comprehensive policy on cancer services. The Lancet Oncology, 7(4), pp 336-346.

Independent Cancer Taskforce. 2015. Achieving world-class cancer outcomes: a strategy for England 2015-2020,

Information Services Division. 2015. Hospital of treatment for selected cancer between 2007-2012 in Scotland.

Jann, B. 2008. nlcheck: Stata module to check linearity assumption after model estimation [Online]. Available: http://ideas.repec.org/ [Accessed June 2016]. 
Jones, A., Haynes, R., Sauerzapf, V., Crawford, S. \& Forman, D. 2010. Geographical access to healthcare in Northern England and post-mortem diagnosis of cancer. Journal of Public Health, 32(4), pp 532-537.

Jones, A., Haynes, R., Sauerzapf, V., Crawford, S., Zhao, H. \& Forman, D. 2008. Travel time to hospital and treatment for breast, colon, rectum, lung, ovary and prostate cancer. European Journal of Cancer, 44(7), pp 992-999.

Khakwani, A., Rich, A. L., Powell, H. A., Tata, L. J., Stanley, R. A., Baldwin, D. R., Duffy, J. P. \& Hubbard, R. B. 2013. Lung cancer survival in England: trends in non-small-cell lung cancer survival over the duration of the National Lung Cancer Audit. Br J Cancer, 109(8), pp 20582065.

Lau, K. K. W., Rathinam, S., Waller, D. A. \& Peake, M. D. 2013. The Effects of Increased Provision of Thoracic Surgical Specialists on the Variation in Lung Cancer Resection Rate in England. Journal of Thoracic Oncology, 8(1), pp 68-72 10.1097/JTO.0b013e3182762315.

Louie, A. V., Palma, D. A., Dahele, M., Rodrigues, G. B. \& Senan, S. 2015. Management of earlystage non-small cell lung cancer using stereotactic ablative radiotherapy: Controversies, insights, and changing horizons. Radiotherapy and Oncology, 114(2), pp 138-147.

Lumley, T., Diehr, P., Emerson, S. \& Chen, L. 2002. The Importance of the Normality Assumption in Large Public Health Data Sets. Annual Review of Public Health, 23(1), pp 151-169.

Matthew G. 1971. Measuring need and evaluating services. In: McLachlan Gordon (ed.) Portfolio for Health; the role and programme of the DHSS in health services research. London: Oxford University Press.

Mungall, I. J. 2005. Trend towards centralisation of hospital services, and its effect on access to care for rural and remote communities in the UK. Rural \& Remote Health, 5(2), pp 8p-8p 1p.

Munro, A. J. 2001. The Calman-Hine report: its causes and consequences. European Journal of Cancer Care, 10(3), pp 213-215.

National Cancer Intelligence Network. 2010. UK Cancer e-Atlas Incidence Statistics [Online]. Available: http://www.ncin.org.uk/cancer information tools/eatlas/guide\#incidence [Accessed November 2014].

National Cancer Intelligence Network. 2014a. Cancer Commissioning Toolkit PCT Profile [Online]. Available: https://www.cancertoolkit.co.uk/Profiles/PCTPublic [Accessed November 2014].

National Cancer Intelligence Network. 2014b. Primary Care Trusts (PCT) Profiles: Meta-data for profile metrics,

National Peer Review Programme, N. E. 2013. My Cancer Treatment [Online]. London. Available: http://www.mycancertreatment.nhs.uk/. 
National Radiotherapy Advisory Group. 2007. Radiotherapy: developing a world class service for England,

NHS. 2015. The NHS Constitution (England).

NHS England. 2012. Securing equity and excellence in commissioning specialised services, (London).

NHS England. 2012-2013. GP Patient Survey PCT results, June 2013. In: Ipsos MORI (ed.).

NHS Wales Informatics Services. Directory of Cancer Services [Online]. Available: http://www.cancerservicesdirectory.wales.nhs.uk/home 2015].

Office of National Statistics. 2013a. Lower Super Output Area Mid-Year Population Estimates [Online]. Available:

https://www.ons.gov.uk/peoplepopulationandcommunity/populationandmigration/populatione stimates/datasets/lowersuperoutputareamidyearpopulationestimates [Accessed June 2015].

Office of National Statistics. 2013b. Population Weighted Centroids Guidance [Online]. ONS. Available: http://www.ons.gov.uk/ons/guidemethod/geography/products/census/spatial/centroids/index.html [Accessed November 2014].

Office of National Statistics. 2013c. Primary Care Organisations Mid Year Population Estimates [Online]. ONS. Available:

https://www.ons.gov.uk/peoplepopulationandcommunity/populationandmigration/populatione stimates/datasets/healthgeographypopulationestimatesprimarycareorganisationsmidyearpop ulationestimates [Accessed June 2015].

Office of National Statistics. 2015a. Postal Geography [Online]. ONS. Available: http://www.ons.gov.uk/ons/guide-method/geography/beginner-s-guide/postal/index.html [Accessed February 2015].

Office of National Statistics. 2015b. Super Output Area [Online]. ONS. Available: http://www.ons.gov.uk/ons/guide-method/geography/beginner-s-guide/census/super-outputareas--soas-/index.html [Accessed February 2015].

Okello, C., Moller, H. \& Davies, E. A. 2011. Reported cancer spending in relation to population characteristics, disease burden and service activity for primary care trusts in South East England. J Public Health (Oxf), 33(3), pp 445-52.

Oliphant, R., Nicholson, G. A., Horgan, P. G., Molloy, R. G., McMillan, D. C. \& Morrison, D. S. 2013. Contribution of surgical specialization to improved colorectal cancer survival. British Journal of Surgery, 100(10), pp 1388-1395.

Oliver, A. \& Mossialos, E. 2004. Equity of access to health care: outlining the foundations for action. $\checkmark$ Epidemiol Community Health, 58(8), pp 655-8. 
Parkin, D. \& Hakulinen, T. 1991. Analysis of Survival. In: Jensen, O., Parkin, D., MacLennan, R., Muir, C. \& Skeet, R. (eds.) Cancer Registration: Principles and Methods. International Agency for Research on Cancer.

Pearce, N. 2000. The ecological fallacy strikes back. Journal of Epidemiology and Community Health, 54(5), pp 326-327.

Round, C., Mee, T., Kirkby, N. F., Cooper, T., Williams, M. V. \& Jena, R. 2013. The Malthus Programme: Developing Radiotherapy Demand Models for Breast and Prostate Cancer at the Local, Regional and National Level. Clinical Oncology, 25(9), pp 538-545.

Walters, S., Quaresma, M., Coleman, M. P., Gordon, E., Forman, D. \& Rachet, B. 2011. Geographical variation in cancer survival in England, 1991-2006: an analysis by Cancer Network. Journal of Epidemiology \& Community Health, 65(11), pp 1044-1052.

Wang, F. 2006. Quantitative Methods and Applications in GIS, Boca Raton: Taylor and Francis.

Wang, F., McLafferty, S., Escamilla, V. \& Lan, L. 2008. Late-Stage Breast Cancer Diagnosis and Health Care Access in Illinois. Professional Geographer, 60(1), pp 54-69.

Williams, R. \& Wright, J. 1998. Epidemiological issues in health needs assessment. In: Wright, J. (ed.) Health Needs Assessment in Practice. London: BMJ Books.

Wright, J., Williams, R. \& Wilkinson, J. 1998. The development and importance of health needs assessment. In: Wright, J. (ed.) Health Needs Assessment in Practice. London: BMJ Books.

Xin, Y. \& Su, X. 2009. Linear Regression Analysis: Theory and Computing, 1st, Singapore: World Scientific Publishing Co. Pte. Ltd. 